\title{
Ascending Aortic B-Cell Lymphoma Masquerading as Intramural Hematoma: A Case Report
}

\author{
Christopher T. Holley ${ }^{* 1}$, John R Spratt ${ }^{1}$, Emil Missov ${ }^{2}$, Elan C Burton ${ }^{3}$ and Sara J Shumway ${ }^{3}$ \\ ${ }^{1}$ University of Minnesota Department of Surgery \\ ${ }^{2}$ University of Minnesota Department of Medicine, Division of Cardiology \\ ${ }^{3}$ University of Minnesota Department of Surgery, Division of Cardiothoracic Surgery
}

${ }^{*}$ Corresponding author: Christopher T. Holley, Department of Surgery, University of Minnesota, USA, E-mail: ctholley@umn.edu

Citation: Christopher T. Holley, John R Spratt, Emil Missov, Elan C Burton, Sara J Shumway (2014) Ascending Aortic B-Cell Lymphoma Masquerading as Intramural Hematoma: A Case Report. J Case Rep Stud 2(1): 104. doi: $10.15744 / 2348-9820.1 .404$

Received Date: January 09, 2014 Accepted Date: February 20, 2014 Published Date: February 24, 2014
Abstract
We report the case of a 74 year old female who presented with imaging concerning for an intramural hematoma of the ascending aorta. Despite multiple imaging modalities consistent with intramural hematoma, operative findings confirmed a peri-aortic lymphoma or what was once lymphoma.

\section{Introduction}

Intramural hematomas (IMH) are a potentially ominous finding due to the concern for progression to frank aortic dissection. Thought to be a variant of aortic dissection without the typical entry/exit site or false lumen flow, IMHs are unpredictable in their behavior and thus challenging to manage. Particularly with ascending IMHs, the high morbidity and mortality associated with a delay in surgical intervention makes timely diagnosis a priority. Most patients present with a sudden onset of chest pain or a mechanism sufficient to cause the suspected injury, such as trauma. Ultrasound and computed tomography (CT) imaging in patients with IMH demonstrate a peri-aortic asymmetric density, either within a focal segment of the aortic wall or completely surrounding the aortic lumen. Intravenous contrast may be used to differentiate intraluminal thrombus and peri-aortic thoracic soft tissue tumors or lymphomas, which will often contrast-enhance. The differential diagnosis in patients with suspected IMH includes: aortic dissection, thrombus, intra-thoracic soft tissue tumors, and lymphoma. Echocardiography, CT, and magnetic resonance imaging (MRI) are useful in better characterizing a suspicious lesion, its location, and guiding management (surgical versus medical). However, despite the advances in imaging technology, limitations remain in diagnosing a suspected aortic IMH or dissection. Complex medical and surgical histories and non-specific symptoms can present further challenges.

In this case, we report a 74 year old female with peri-aortic lymphoma who presented with imaging features most suggestive of an ascending aortic IMH. Our patient presented without symptoms. Multiple imaging modalities demonstrated features more commonly associated with IMH than lymphoma. Focal, asymmetric wall thickening seen both on ultrasound and CT/MRI were most consistent with IMH. Features typically seen with thoracic lymphomas, such as significant lymphadenopathy, pleural effusions, and contrast enhancement, were absent.

\section{Case Report}

A 74 year old female with a remote past medical history of T-Cell lymphoma, in remission for 25 years, and a recent diagnosis of B-Cell lymphoma, was admitted to the hospital after an incidental finding of focal, asymmetric aortic wall thickening. An ascending intramural hematoma was suspected based on the appearance from an elective CT scan performed for staging of her B-Cell lymphoma. Transthoracic echocardiography (TTE) (Figure 1) and transesophageal echocardiography (TEE) were performed to confirm the location and further characterize the lesion and demonstrated circumferential, but asymmetric soft tissue thickening surrounding the aorta. MRI (Figure 2) imaging demonstrated eccentric wall thickening with degradation product, suggesting hemorrhage or hematoma. All imaging modalities demonstrated thickening of the ascending aorta most consistent with intramural hematoma. No clear dissection planes or entry/exit sites were able to be identified. Cardiothoracic surgery was consulted and the patient was transferred to the intensive care unit (ICU) for aggressive blood pressure control. The patient had been asympto- 
matic, and denied chest pain, shortness of breath, dyspnea on exertion, or neurologic symptoms.

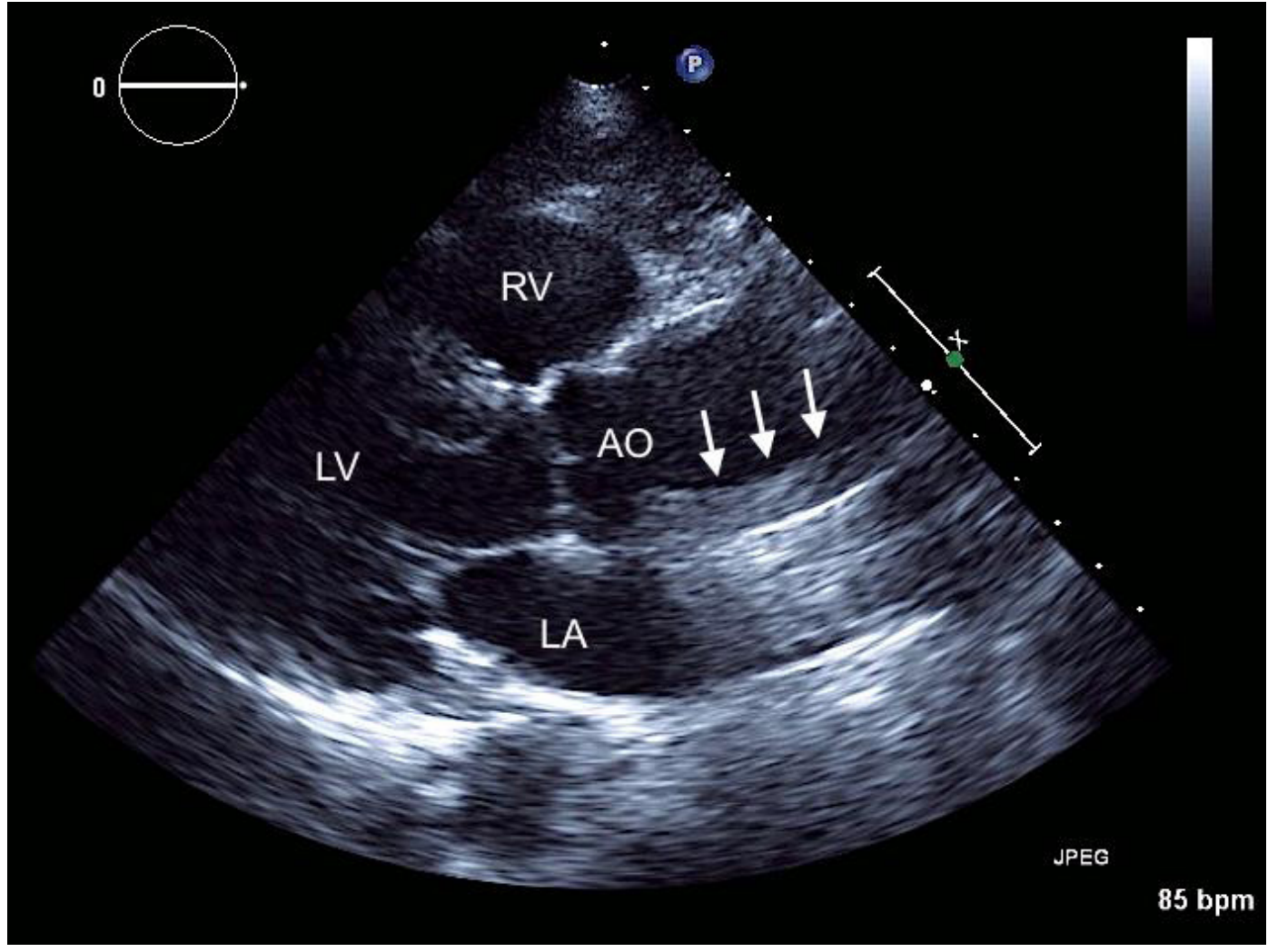

Figure 1: Transthoracic echocardiogram in the parasternal long axis view identifies the left ventricle (LV), right ventricle (RV), left atrium (LA), and proximal aorta (AO). The aortic wall is abnormally thick (arrows) consistent with intramural hematoma.

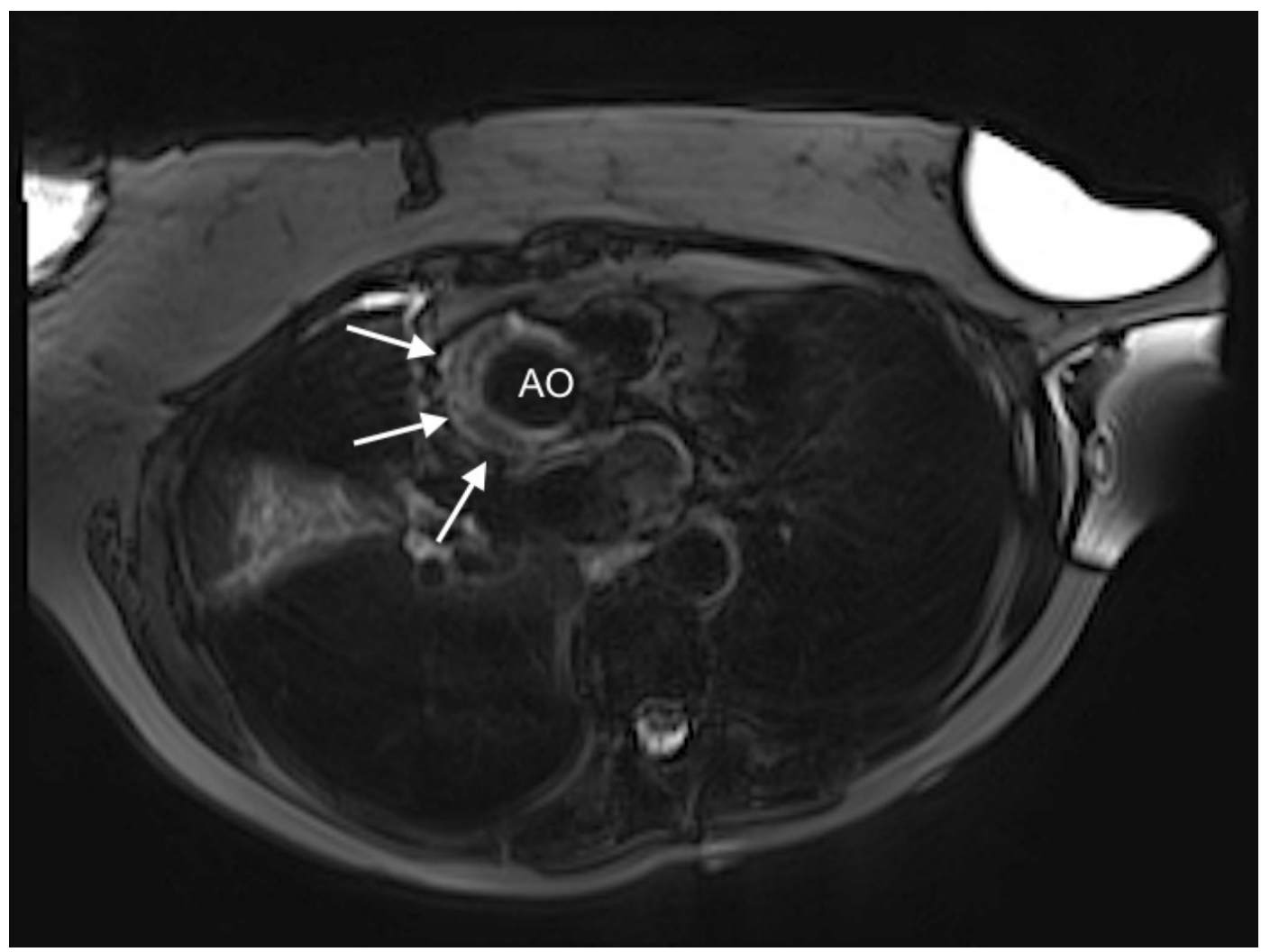

Figure 2: MRI post-contrast T1 fat-saturated weighted image. Eccentric wall thickening of the aorta (AO) with degradation products consistent with hemorrhage or hematoma (arrows). 
Due to concern for rupture, our patient was taken to the operating room for planned resection and repair of the ascending aorta. In the operating room, however, an absence of blood was noted in the pericardial space and the ascending aorta appeared to be encased in thick interstitial tissue consistent with periaortic lymphoma from approximately $1 \mathrm{~cm}$ distal to the aortic annulus to 5 $\mathrm{cm}$ proximal to the takeoff of the innominate artery. Intra-operative biopsies were obtained and sent to pathology to confirm the suspected diagnosis. Final pathology did not confirm active lymphoma but a mixed inflammatory infiltrate, likely residual tissue from her previously treated lymphoma. The planned operation was terminated, and the patient was taken back to the ICU for monitoring. The remainder of her hospital course was unremarkable. She recovered as expected and was able to be discharged to her home on postoperative day four. Over one year later, she remains asymptomatic.

\section{Discussion}

This case describes a patient with a known history of lymphoma, who presented with radiologic evidence of intramural hematoma. Intrathoracic lymphoma most commonly presents with pleural effusions, thoracic lymphadenopathy or pulmonary masses. Extrapleural involvement is an uncommon radiographic feature [1,2]. Case reports of periaortic lymphoma mimicking descending aortic aneurysm [3], or causing descending aortic dissection [4], have been described.

Intramural hematomas are most frequently seen in patients who present with acute aortic syndromes. Their management, however, remains controversial. The natural evolution of an IMH is poorly understood, but is thought to be secondary to vasa vasorum rupture, causing localized hematoma within the media of the aortic wall. Ongoing bleeding or resorption of this blood can eventually result in frank aortic dissection and/or aortic rupture. Previous authors have quoted a mortality of 33-60\% for patients presenting with ascending aortic intramural hematomas [5] and a progression of IMH to frank aortic dissection or aortic rupture in 20-47\% of patients [6]. Regression of the IMH can occur but cannot be predicted and is much less common [7]. Because of this, early surgical intervention has been advocated as the standard of care for patients found to have IMH of the ascending aorta radiographically [8].

Some have advocated for the role of medical management in selected patients, even with IMH of the ascending aorta [9]. The high incidence of persistence or progression of the IMH (81\% in one study [10]) and a lack of reliable factors predicting either early or late progression have led most to recommend aggressive, early surgical management of IMH involving the ascending aorta. IMH of the descending aorta, however, have a much lower mortality and risk of progression and can be managed medically in selected patients[5].

In our patient, the recommendation for surgical intervention for presumed IMH despite her lack of symptoms was based primarily on this concern for progression to dissection or rupture, given its imaging findings and location. In fact, a retrospective review demonstrated that more than $6 \%$ of patients with acute aortic dissection may present without chest or back pain[11]. In addition, it was shown that painless acute aortic dissection was associated with a delay in diagnosis and higher in hospital mortality, highlighting the importance of timely diagnosis and treatment.

Previous case reports of tumors mimicking aortic dissections or aneurysms have been reported [12-14], although most have been prior to the availability of high-resolution CT or MRI. In cases with unusual presenting symptoms or an unreliable history confirmatory imaging is critical to establishing the correct diagnosis and avoiding unnecessary surgery. Despite reported specificities of up to $100 \%$ for modern imaging modalities in the diagnosis of aortic dissection [15], case reports of false positive CT scans later shown to be artifact on confirmatory imaging, exist [16]. In one case, exploratory thoracotomy was required to definitively differentiate between suspected neoplastic disease and aortic dissection, despite multiple CT and MRI imaging studies [17].

Imaging features differentiating IMH from periaortic lymphoma, or other causes, have been the lack of contrast enhancement in IMH, whereas lymphoma typically demonstrates enhancement on CT and MRI. However, in this case, the lack of contrast enhancement despite CT angiography (CTA) and magnetic resonance angiography (MRA) did little to identify the cause of the aortic mass. In one paper, fludeoxyglucose-positron emission tomography (FDG-PET) CT was used to confirm suspected peri-aortic lymphoma in a man presenting after a motor vehicle accident with chest pain and imaging concerning for IMH [18].

\section{Conclusion}

Diagnosing a periaortic mass can be difficult despite the widespread availability of complex and high resolution imaging modalities. In the setting of conflicting clinical and radiographic pictures, and complex past medical and surgical histories, there remains a concern for progression without timely intervention. While neoplastic disease surrounding the descending thoracic and abdominal aorta have been known to occur, and mediastinal tumors have been known to cause symptoms that mimic aortic dissection clinically, we are unaware of any cases of neoplastic disease causing radiographic features which mimic intramural hematoma or type A dissection. 
In our patient, despite her known lymphoma and lack of an acute aortic syndrome on presentation, CT and MRI imaging demonstrated an asymmetric periaortic mass which lacked contrast enhancement, making her diagnosis challenging. When considering the differential diagnosis of an ascending periaortic mass, life threatening causes such as intramural hematoma, aortic dissection, and aortic rupture must be ruled out. Confirmatory imaging may help minimize false positive imaging findings in patients where the diagnosis remains unclear but, as in this case, may not definitively rule in or rule out a life threatening disease process. A lack of chest or back pain occurs in a small, but substantial subset of patients presenting with aortic dissections and can lead to a delay in diagnosis and poor outcomes.

It remains the opinion of the authors that suspected or confirmed ascending aortic intramural hematoma should be managed similarly to type A aortic dissections, with timely surgical intervention. Primary or secondary periaortic lymphoma must be considered, particularly in a patient with a known history of malignancy who presents without clinical symptoms. In a clinically stable, asymptomatic patient, FDG-PET/CT may help to differentiate between IMH and lymphoma.

\section{References}

1) Celikoglu F, Teirstein AS, Krellenstein DJ, Strauchen JA (1992) Pleural effusion in non-Hodgkin's lymphoma. Chest 101: 1357-60.

2) Aquino SL, Chen MY, Kuo WT, Chiles C (1999) The CT appearance of pleural and extrapleural disease in lymphoma. Clin Radiol 54: 647-50.

3) Carro C, Camilleri L, Garcier JM, De Riberolles C (2004) Periaortic lymphoma mimicking aortic aneurysm. Eur J Cardiothorac Surg 25: 1126.

4) Lotto AA, Kendall SW, Hartley R, Walker P (2007) A case of a periaortic lymphoma presenting with the features of descending thoracic aorta dissection. Br J Radiol 80: 30-2.

5) Evangelista A, Mukherjee D, Mehta RH, O'Gara PT, Fattori R, et al. (2005) Acute intramural hematoma of the aorta; A mystery in evolution. Circulation 111: 1063-70.

6) Nienaber CA, Eagle KA (2003) Aortic dissection frontiers in diagnosis and management, part I: from etiology to diagnostic strategies. Circulation 108: 628-35. 7) Von Kodolitsch Y, Csosz SK, Koschyk DH, Schalwat I, Loose R, et al. (2003) Intramural hematoma of the aorta: predictors of progression to dissection and rupture. Circulation 107: 1158-63.

8) Neri E, Capannini G, Carone E, Diciolla F, Sassi C (1999) Evolution toward dissection of an intramural hematoma of the asceding aorta. Ann Thorac Surg 68: $1855-6$.

9) Song JK, Kim HS, Kang DH, Lim TH, Song MG, et al. (2001) Different clinical features of aortic intramural hematoma versus dissection involving the ascending aorta. J Am Coll Cardiol 37: 1604-10.

10) Tittle SL, Lynch RJ, Cole PE, Singh HS, Rizzo JA, et al. (2002) Midterm follow-up of penetrating ulcer and intramural hematoma of the aorta. J Thorac Cardiovasc Surg 123: 1051-9.

11) Park SW, Hutchison S, Mehta RH, Isselbacher EM, Cooper JV, et al. (2004) Association of Painless Acute Aortic Dissection With Increased Mortality. Mayo Clin Proc 79: 1252-7.

12) Eagle KA, Quertermous T, Kritzer GA, Newell JB, Dinsmore R, et al. (1986) Spectrum of conditions initially suggesting acute aortic dissection but with negative aortograms. Am J Cardiol 57: 322-6.

13) Margolis JA, Cohn RA, Griffin JP, Flanagan WH, Person BT (1980) Oat cell carcinoma presenting as a pseudoaneurysm. South Med J 73: 1087-9.

14) Shin MS, Ho KJ, Kirklin JW, Sears NJ (1985) Metastatic mediastinal neoplasm masquerading as aortic dissection: a skip sign on computed tomography for their distinction. J Comput Tomogr 9: 299-303.

15) Barbant SD, Eisenberg MJ, Schiller NB (1992) The diagnostic value of imaging techniques for aortic dissection. Am Heart J 124: 541-3.

16) Firstenberg MS, Crestanello JA, Sai-Sudhakar CB, Sirak JH, Sun B (2008) Ascending aortic dissection: look again before you leap. Ann Thorac Surg 85: 1782-4.

17) Kazerooni EA, Williams DM, Michael Deeb G (1992) Thoracic periaortic lymphoma mimicking aortic dissection. AJR Am J Roentgerol 159: 705-7.

18) Lu MT, Millstine J, Menard M, Rybiki F, Viscomi S (2006) Periaortic lymphoma as a mimic of posttraumatic intramural hematoma. Emerg Radiol 13: 35-8.

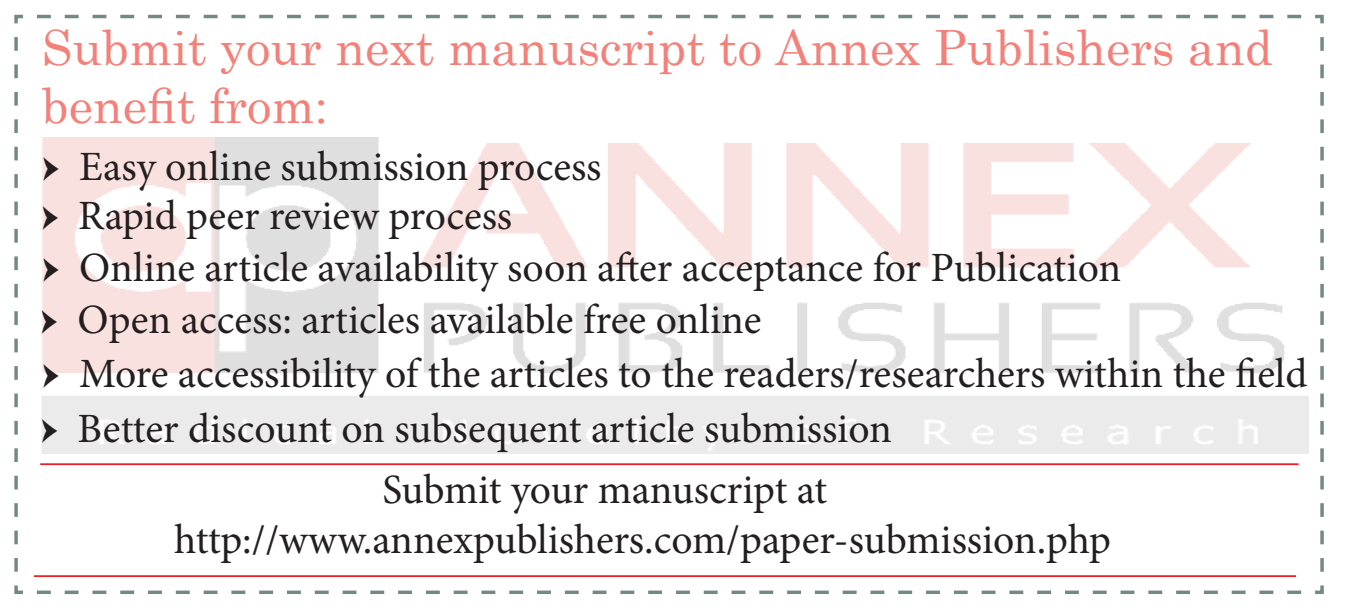

